\title{
High-resolution in vivo imaging of human locus coeruleus by Magnetization Transfer MRI at 3T and 7T
}

Citation for published version (APA):

Priovoulos, N., Jacobs, H. I. L., Ivanov, D., Uludag, K., Verhey, F. R. J., \& Poser, B. A. (2018). Highresolution in vivo imaging of human locus coeruleus by Magnetization Transfer MRI at 3T and 7T. Neuroimage, 168, 427-436. https://doi.org/10.1016/j.neuroimage.2017.07.045

Document status and date:

Published: 01/03/2018

DOI:

10.1016/j.neuroimage.2017.07.045

Document Version:

Publisher's PDF, also known as Version of record

Document license:

Taverne

Please check the document version of this publication:

- A submitted manuscript is the version of the article upon submission and before peer-review. There can be important differences between the submitted version and the official published version of record.

People interested in the research are advised to contact the author for the final version of the publication, or visit the DOI to the publisher's website.

- The final author version and the galley proof are versions of the publication after peer review.

- The final published version features the final layout of the paper including the volume, issue and page numbers.

Link to publication

\footnotetext{
General rights rights.

- You may freely distribute the URL identifying the publication in the public portal. please follow below link for the End User Agreement:

www.umlib.nl/taverne-license

Take down policy

If you believe that this document breaches copyright please contact us at:

repository@maastrichtuniversity.nl

providing details and we will investigate your claim.
}

Copyright and moral rights for the publications made accessible in the public portal are retained by the authors and/or other copyright owners and it is a condition of accessing publications that users recognise and abide by the legal requirements associated with these

- Users may download and print one copy of any publication from the public portal for the purpose of private study or research.

- You may not further distribute the material or use it for any profit-making activity or commercial gain

If the publication is distributed under the terms of Article $25 \mathrm{fa}$ of the Dutch Copyright Act, indicated by the "Taverne" license above, 


\title{
High-resolution in vivo imaging of human locus coeruleus by magnetization transfer MRI at 3T and 7T
}

\author{
Nikos Priovoulos ${ }^{\mathrm{a}, *}$, Heidi I.L. Jacobs ${ }^{\mathrm{a}, \mathrm{b}, \mathrm{c}}$, Dimo Ivanov ${ }^{\mathrm{b}}$, Kâmil Uludağ ${ }^{\mathrm{b}}$, Frans R.J. Verhey ${ }^{\mathrm{a}}$, \\ Benedikt A. Poser ${ }^{b}$ \\ a Alzheimer Center Limburg, School for Mental Health and Neuroscience, Faculty of Health, Medicine and Life Sciences, Maastricht University, Maastricht, The \\ Netherlands \\ ${ }^{\mathrm{b}}$ Department of Cognitive Neuroscience, Faculty of Psychology and Neuroscience, Maastricht University, Maastricht, The Netherlands \\ ${ }^{\mathrm{c}}$ Department of Radiology, Division of Nuclear Medicine and Molecular Imaging, Massachusetts General Hospital/Harvard Medical School, Boston, MA, USA
}

\section{A R T I C L E I N F O}

\section{Article history:}

Received 9 December 2016

Received in revised form

19 July 2017

Accepted 20 July 2017

Available online 22 July 2017

\section{Keywords:}

Locus coeruleus

Magnetization transfer

Neuromelanin

Brainstem

Norepinephrine

7T

3T

MRI

\begin{abstract}
A B S T R A C T
Locus Coeruleus (LC) is a neuromelanin-rich brainstem structure that is the source of noradrenaline in the cortex and is thought to modulate attention and memory. LC imaging in vivo is commonly performed with a 2D $\mathrm{T}_{1}$-weighted Turbo Spin Echo (TSE) MRI sequence, an approach that suffers from several drawbacks at 3T, including long acquisition times and highly anisotropic spatial resolution. In this study, we developed a high-resolution Magnetization Transfer (MT) sequence for LC imaging at both 7T and 3T and compared its performance to a TSE sequence. Results indicate that LC imaging can be achieved with an MT sequence at both 7 and 3T at higher spatial resolution than the 3T TSE. Furthermore, we investigated whether the currently disputed source of contrast in the LC region with a TSE sequence relates to MT effects or shortened $\mathrm{T}_{1}$ and $\mathrm{T}_{2}{ }^{*}$ due to increased iron concentration. Our results suggest that the contrast in the LC area relates to MT effects. To conclude, in this study we managed to image the LC, for the first time, at $7 \mathrm{~T}$ and at an increased resolution compared to the current state-of-the-art. Imaging the LC is highly relevant for clinical diagnostics as structural tissue properties of the LC may hold promise as a biomarker in neurodegenerative diseases.
\end{abstract}

(c) 2018 Published by Elsevier Inc.

\section{Introduction}

The locus coeruleus (LC) is a small (approximately $15 \mathrm{~mm}$ long and $2 \mathrm{~mm}^{2}$ thick in humans), rod-shaped nucleus, located deep in the pons along the lateral edge of the fourth ventricle (Baker et al., 1989; Fernandes et al., 2012; German et al., 1988). The LC is the source of noradrenaline in the cortex and is thought to modulate sleep, arousal (Berridge and Waterhouse, 2003) and several cognitive functions, including memory (Mather et al., 2015; O'Carroll et al., 1999; Sterpenich et al., 2006) and attention (Aston-Jones et al., 2000). Furthermore, LC atrophy has been related to Alzheimer's disease (AD) (Busch et al., 1997; Marcyniuk et al., 1986), Parkinson's disease (PD), depression (Weiss et al., 1994), Post-Traumatic Stress Disorder (PTSD) (Hendrickson and Raskind, 2016) and cognitive reserve in ageing (Clewett et al., 2016). Interest in imaging the LC in vivo and in evaluating its potential as a biomarker has been omnipresent, but our ability to achieve this has been limited by the

\footnotetext{
* Corresponding author. Alzheimer Center Limburg, Department of Psychiatry and Neuropsychology, UNS40 box 34, P.O. Box 616, 6200 MD Maastricht, The Netherlands.

E-mail address: nikos.priovoulos@maastrichtuniversity.nl (N. Priovoulos).
}

poor spatial resolution and contrast provided by standard MRI methods. It is the aim of this study to demonstrate and optimize in vivo LC imaging using 7T MRI, and to translate this back to the 3T environment to facilitate widespread clinical applications.

Sasaki et al. (2006) were the first to report a hyperintensity in the $\mathrm{LC}$ region with a $\mathrm{T}_{1}$-weighted Turbo Spin Echo (TSE) sequence at $3 \mathrm{~T}$ (Sasaki et al., 2006). While TSE is currently the method of choice for LC imaging in vivo, it suffers from several disadvantages. First, LC-TSE acquisitions necessitate long scanning times (the acquisition time in recent papers employing LC imaging is $12 \mathrm{~min}$ (Castellanos et al., 2015; Ohtsuka et al., 2013; Takahashi et al., 2015)), due to low signalto-noise ratio (SNR). This may be problematic for populations that are challenging to image because an extended scan duration poses an unacceptable burden to them, or they find it difficult to remain still for the duration of a measurement (Reuter et al., 2015). Second, the numerous radiofrequency (RF) pulses used in a TSE sequence result in high power deposition, which makes its application to LC imaging in 7T highly impractical. The more than four-fold increased Specific Absorption Rate (SAR) would require use of long repetition times (TR) (Uğurbil, 2014) and hence drastically prolonged scan durations, which however is incompatible with the desire to keep scans short; especially, if the objective is to translate the field strength advantage of $7 \mathrm{~T}$ 
into higher spatial resolution. Most previous studies have applied TSE as a 2-dimensional (2D) sequence with anisotropic resolution and rather large slice thickness of $2.5-3 \mathrm{~mm}$ to achieve sufficient SNR for any depiction at all, and to keep scan time within acceptable limits (Castellanos et al., 2015; Ohtsuka et al., 2013; Takahashi et al., 2015). While TSE has been employed in several studies, its disadvantages have limited the widespread use and clinical utility of LC imaging even at 3T.

Anatomical precision of the LC contrast in $\mathrm{T}_{1}$-weighted TSE was recently confirmed as it spatially matches the location of the LC cells that contain neuromelanin (NM) (Keren et al., 2015). NM is a macromolecule that is a by-product of noradrenaline and dopamine synthesis (Smythies, 1996), and is known to bind iron molecules in areas such as the substantia nigra (SN) (Enochs et al., 1994; Zecca and Swartz, 1993). It has therefore been hypothesized that the $\mathrm{T}_{1}$-TSE contrast may relate to increased iron concentration in the LC cells (Sasaki et al., 2006). Gradient echo (GRE) based MR techniques that are particularly sensitive to iron, such as $\mathrm{T}_{2}{ }^{*}$ mapping (Haacke et al., 2005; Langkammer et al., 2010) and susceptibility weighted imaging (Liu et al., 2015), have thus been suggested as a possible alternative for imaging NM (Langley et al., 2015; Silvy Collin et al., 2014). Contrary to this hypothesis, however, Zucca et al. (2006) reported low levels of iron deposition in LC (Zucca et al., 2006). Another possible source of the LC contrast in $\mathrm{T}_{1}$-TSE is incidental magnetization transfer (MT) (Keren et al., 2015), since the high number of RF pulses in a 2D TSE scan results in off-resonance magnetization saturation (Dixon et al., 1990).

MT contrast can be explained by considering a two-pool proton model, with a pool of protons in free water and a pool of protons that are bound in surrounding macromolecules (Henkelman et al., 1993). $\mathrm{RF}$ pulses at an off-resonance frequency are applied to preferentially saturate the spins of the "bound" pool, and this magnetization transfers to the "free" pool at a tissue specific exchange rate. This may create an MR detectable MT contrast between NM-rich regions and the surrounding brain tissue. LC contrast has been reported in two studies that employed dedicated off-resonant RF pulses combined with a GRE readout (Chen et al., 2014; Nakane et al., 2008). This indicates that MT effects are another likely source of the $\mathrm{T}_{1}$-TSE contrast in the $\mathrm{LC}$ that may act independently to $\mathrm{T}_{1}$-shortening due to iron deposition. There is currently great research interest in developing a quantitative estimation method for LC atrophy, for which a better understanding of the source of LC contrast is of vital importance. To the best of our knowledge, however, no previous study has compared different MR contrasts in the LC region.

Under the hypothesis that LC contrast is driven by magnetization transfer, a GRE-readout interleaved with a pre-saturation pulse may be an efficient method of LC imaging, with lower SAR than TSE, which can be regarded as the current 'gold standard'. Moreover, a longer pre-saturation, consisting of a train of MT pulses, interleaved with a fast readout before recovery, such as with a segmented 3-dimensional (3D) turbo FLASH (TFL) (Hennig et al., 1986), can result in a more efficient and faster approach compared to a standard MT-GRE (Mougin et al., 2010). The reduced acquisition time becomes a major advantage for the high spatial resolutions achievable at UHF, crucial for small subcortical structures, such as LC. In addition to SNR, a MT-based contrast may benefit at 7T from the bigger chemical shift between the macromolecular pool and the free water pool as well as the slower recovery from saturation due to longer $T_{1} s$ (Mougin et al., 2010).

The aim of this study was to optimize LC imaging for 7T and to demonstrate its feasibility. To this end, we developed an MTweighted TFL sequence for high-resolution LC imaging and compared its performance to the TSE at both 3T and 7T. Furthermore, we explored if the LC contrast commonly observed in the TSE relates to MT effects or shortened $\mathrm{T}_{2}{ }^{*}$ or $\mathrm{T}_{1}$ potentially due to increased iron deposition in the LC.

\section{Methods}

\subsection{Data acquisition}

MR data from 6 young healthy participants (2 females; age range $=21-31$; mean $\pm S D=25.6 \pm 4.1$ years) were acquired on a Siemens Magnetom 7T scanner with a 32-channel head coil (Nova Medical, Wilmington, MA, USA), and a Siemens 3T Prisma Fit with a 64-channel head/neck coil (Siemens Healthineers, Erlangen, Germany). All participants provided written informed consent. Approval of the experimental protocol was obtained from the local ethical committee of the Faculty of Psychology and Neuroscience at Maastricht University. Since depression has been linked to LC atrophy (Klimek et al., 1997), all participants were asked prior to the scanning session to fill in the Hamilton Rating Scale for Depression (Hamilton, 1960). All participants scored within the normal range (range $=0-3$; mean $\pm \mathrm{SD}=1.2 \pm 1.3$ ).

\subsubsection{Magnetization transfer-weighted sequence piloting}

A magnetization transfer-weighted turbo flash (MT-TFL) sequence was developed in-house, similar to the MT sequence described by Mougin and colleagues (Mougin et al., 2013). Our sequence consisted of an adjustable train of $10 \mathrm{~ms}$ long off-resonant Gaussian sinc pulses (bandwidth $=250 \mathrm{~Hz}$ ), which was followed by a Cartesian readout. Each TFL readout performed the partition encoding ( $\mathrm{kz}$-phase encoding) in a centre-out fashion, whereas the in-plane (ky) phase encoding was performed in a low-to-high linear fashion. Sampling the center of k-space first ensured maximum MT-weighting (Mougin et al., 2010). No parallel undersampling was employed, with 6/8 phase and slice partial Fourier.

In the piloting phase of this study, a set of experiments was conducted to optimize the sequence for LC imaging at $7 \mathrm{~T}$ on one healthy adult volunteer. First, for the target imaging protocol with approximately $500 \mathrm{~ms}$ TFL readout (see detailed parameters in Table 1), we evaluated two different MT saturation preparation trains by calculating the SNR and contrast-to-noise ratio (CNR) in the LC as shown in the Supplementary material: (1) 8 pulses of amplitude $4.50 \mu \mathrm{T}(0.37 \mu \mathrm{T}$ continuous wave power equivalent (CWPE) calculated as shown in (Ramani et al., 2002)) and (2) 20 pulses of $3.37 \mu \mathrm{T}(0.46 \mu \mathrm{T}$ CWPE). The range of pulse sequence parameters tested was chosen based on the report of Mougin and colleagues (Mougin et al., 2013). The MT amplitude in each case was chosen empirically as the maximum amplitude that was within SAR constraints for a $55 \mathrm{~kg}$ participant without an extension of the repetition time (TR); this allowed the same protocol to be used on all participants. The frequency offset was fixed to $4.03 \mathrm{ppm}$. For the 20-pulse train which performed best (S-Table 1, S-Fig. 1), we proceeded to vary the off-resonance frequency at $4.03 \mathrm{ppm}, 6.72 \mathrm{ppm}$ and $11.76 \mathrm{ppm}$, based on z-spectrum reports of white and grey matter (Mougin et al., 2013) and the neuromelanin-rich SN (Trujillo et al., 2015). A frequency offset of $6.72 \mathrm{ppm}$ was chosen as optimal (S-Table 2, S-Fig. 2). A schematic of the sequence is shown in S-Fig. 3.

\subsubsection{Tesla data acquisition}

We performed a MT-TFL 2-average scan using a MT saturation of 20 pulses of $3.37 \mu \mathrm{T}(0.46 \mu \mathrm{T}$ CWPE $)$ at a frequency offset of $6.72 \mathrm{ppm}$. To further examine the performance of the MT-TFL scan, we also acquired a single acquisition MT-TFL with the same parameters. To help establish whether the LC contrast reported in the MR experiments is due to MT, we also acquired a TFL with the MT pulses turned off. To examine the relation between the LC contrast reported in MR experiments and iron concentration, we also acquired a multi-echo-gradient echo sequence (ME-GRE). Finally, to calculate an $R_{1}$ map and facilitate between-scanner registration, we acquired a Magnetization Prepared 2 Rapid Acquisition Gradient 
Table 1

Parameters of MR sequences performed.

\begin{tabular}{|c|c|c|c|c|c|c|c|c|c|c|}
\hline Dataset & Sequence & Averages & $\begin{array}{l}\text { Flip angle }(\mu \mathrm{T}) \text { of } \\
\text { MT saturation }^{\mathrm{A}}\end{array}$ & $\begin{array}{l}\text { Repetition } \\
\text { time (ms) }\end{array}$ & Echo time (ms) & $\begin{array}{l}\text { Turbo } \\
\text { factor }\end{array}$ & $\begin{array}{l}\text { Flip angle } \\
\text { (degree) }\end{array}$ & Voxel size $\left(\mathrm{mm}^{3}\right)$ & $\begin{array}{l}\text { Number } \\
\text { of slices }\end{array}$ & $\begin{array}{l}\text { Acquisition } \\
\text { time (min) }\end{array}$ \\
\hline \multirow[t]{5}{*}{$7 \mathrm{~T}$} & MT - TFL 2-average & 2 & 0.46 & 823 & 4.08 & 38 & $8^{\circ}$ & $0.4 \times 0.4 \times 0.5$ & 60 & $9: 16$ \\
\hline & MT - TFL 1-average & 1 & 0.46 & 823 & 4.08 & 38 & $8^{\circ}$ & $0.4 \times 0.4 \times 0.5$ & 60 & $4: 38$ \\
\hline & TFL (no saturation) & 1 & - & 823 & 4.08 & 38 & $8^{\circ}$ & $0.4 \times 0.4 \times 0.5$ & 60 & $3: 02$ \\
\hline & ME-GRE & 1 & - & 37 & $\begin{array}{l}2.75 / 7.43 / 14.86 / \\
23.39\end{array}$ & & $11^{\circ}$ & $0.4 \times 0.4 \times 0.5$ & 60 & $9: 32$ \\
\hline & MP2RAGE & 1 & - & 5000 & 2.47 & & $5^{\circ} / 3^{\circ}$ & $0.7 \times 0.7 \times 0.7$ & 240 & $9: 42$ \\
\hline \multirow[t]{3}{*}{$3 \mathrm{~T}$} & MT - TFL 4-average & 4 & 0.82 & 508 & 3.92 & 23 & $8^{\circ}$ & $0.4 \times 0.4 \times 1$ & 30 & $11: 28$ \\
\hline & TSE & 5 & - & 600 & 14 & 2 & $90^{\circ}$ & $0.4 \times 0.4 \times 2$ & 14 & $11: 47$ \\
\hline & MPRAGE & 1 & - & 5000 & 2.21 & & $9^{\circ}$ & $1 \times 1 \times 1$ & 256 & $5: 05$ \\
\hline $\begin{array}{l}\text { Previously } \\
\text { acquired }\end{array}$ & ME-GRE & 1 & - & 37 & $\begin{array}{l}2.65 / 7.37 / 13.10 / \\
21.11\end{array}$ & & $11^{\circ}$ & $0.5 \times 0.5 \times 0.5$ & 80 & $8: 33$ \\
\hline 7T ME-GRE & MP2RAGE & 1 & - & 5000 & 2.47 & & $5^{\circ} / 3^{\circ}$ & $0.7 \times 0.7 \times 0.7$ & 240 & $9: 42$ \\
\hline
\end{tabular}

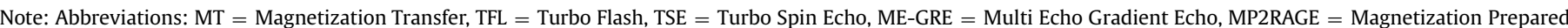

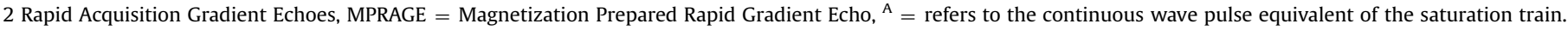
The saturation sequence consisted of 20 pulses at $6.72 \mathrm{ppm}$ off-resonance with bandwidth $=250 \mathrm{~Hz}$.

Echoes (MP2RAGE) sequence (Marques et al., 2010). For details of the MR sequences parameters, we refer to Table 1. For all the sequences except the MP2RAGE, the field-of-view (FOV) was placed approximately perpendicular to the pons and covered the area between the inferior colliculus and the inferior border of the pons. The order of the acquisitions was permuted pseudo-randomly between participants. Due to an acquisition error, a MT-TFL 2-average was not acquired from one participant, leaving 5 acquisitions for analysis. In one participant, we acquired the MT-TFL 2-average three times (one in a separate session) for a reproducibility analysis.

\subsubsection{Previously acquired 7 Tesla multi-echo GRE}

To validate our conclusions about $R_{2}{ }^{*}$ and $R_{1}$ increase in the LC on an independent dataset, we included a dataset $(\mathrm{N}=18)$ acquired from healthy individuals at 7T at an earlier date. This dataset consisted of high-resolution multi-echo GRE and MP2RAGE acquisitions. FOV was placed as described in the 7T acquisition section. Details can be found in Table 1.

\subsubsection{Tesla data acquisition}

At 3T, we performed a 4-average MT-TFL acquisition with an MT saturation of 20 pulses of $6.75 \mu \mathrm{T}(0.82 \mu \mathrm{T}$ CWPE, chosen empirically as the maximum value that was within SAR constraints for the given repetition time (TR) for a $55 \mathrm{~kg}$ participant. Note that this is higher than the pulse amplitude employed at 7T; we chose this to maximize MT-weighting) at a frequency offset of $6.72 \mathrm{ppm}$. In addition, we acquired a $\mathrm{T}_{1}$-weighted TSE sequence similar to the LC imaging protocol employed in previous studies (Keren et al., 2009; Sasaki et al., 2006; Takahashi et al., 2015). Both sequences had a matched in-plane resolution and a comparable acquisition time to the MT-TFL and TSE employed at the 7T. We also obtained a Magnetization Prepared Rapid Acquisition Gradient Echo (MPRAGE) sequence to facilitate registration across field strengths. The detailed parameters of the MR sequences performed at 3T are listed in Table 1. The FOV, for all sequences except the MPRAGE, was placed perpendicularly to the pons to minimize partial volume effects but was otherwise similar to the one used in the 7T acquisitions. Similar to the 7T data acquisition, the order of the scan acquisitions was permuted pseudorandomly and, in one participant, we acquired the TSE three times (one in a separate session). Due to an acquisition error, we did not acquire a MT-TFL from one participant, leaving 5 acquisitions for analysis.

\subsection{Processing}

\subsubsection{Registrations}

First, the brainstem specific images were registered to each other with a rigid body transform from the Advanced Normalization Tools package (ANTS) (Avants et al., 2011) and to the full brain $T_{1}$ images using a boundary based registration (BBR) from the FreeSurfer package (version 6.0 beta). Subsequently, the pons was automatically segmented in the full brain $T_{1}$ images using FreeSurfer (Iglesias et al., 2015). The pons segmentations of the 3T and the 7T were registered to each other employing a 7 degrees-of-freedom (dof) transform with ANTS and the concatenation of BBR and 7-dof transforms was used to project the brainstem specific images from 3T to 7T. For the previously acquired 7T ME-GRE dataset, we registered the full brain $\mathrm{T}_{1}$ image to the MNI space employing a diffeomorphic transform (ANTS (Avants et al., 2011; Klein et al., 2009)).

\subsubsection{Locus coeruleus segmentation}

We then segmented the LC in the 3T TSE, 3T MT-TFL and 7T MTTFL images. Following consultation with a neuroanatomical expert (WB), the superior and inferior boundaries of the $\mathrm{LC}$ were defined in the 7T $\mathrm{T}_{1}$-weighted image. A superior boundary was defined by an axial plane aligned to the inferior border of the inferior colliculus and the mammillary body. An inferior boundary was defined by identifying a plane at the level of the lateral recess of the 4th ventricle (Fig. 1A). The superior and inferior boundaries were projected to all LC images. The hyperintense voxels at the lateral edge of the 4 th ventricle between these boundaries (Fig. 1B) were selected on each axial slice with a $4 \times 4$ voxel ROI, by two researchers independently (NP and LP), starting from the slice on which the hyperintensity was most obvious. The inter-rater reliability was examined based on the Jaccard ratio $\left(J=\frac{L C_{1} \cap L C_{2}}{L C_{1} \cup L C_{2}}\right.$ where $L_{1}$ is the segmentation from researcher 1 and $\mathrm{LC}_{2}$ is the segmentation from researcher 2 ) (Table 2 ). The voxels selected by both researchers were chosen to form the initial LC ROIs. The initial LC ROIs were visually checked by the researchers to avoid contamination from voxels of the 4 th ventricle (Fig. 1C). A $3 \times 3$ voxel $\left(\sim 1.2 \times 1.2 \mathrm{~mm}^{2}\right)$ cross centered on the highest intensity voxel within each slice of each initial LC ROI was created to form the final LC ROI (Fig. 1D) similar to the most common LC segmentation approach (Clewett et al., 2016; Keren et al., 2009). Furthermore, a reference ROI (to be used for contrast comparisons) was drawn manually in the pontine tegmentum (PT) in the space of the 7T $\mathrm{T}_{1}$-weighted image (to account for different FOV orientations between scans) as follows: first a plane $7 \mathrm{~mm}$ inferior to the superior boundary for the LC was chosen. Then the apex of the 4 th ventricle was defined as the dorsal edge of the ROI and a $4 \times 4 \times 2 \mathrm{~mm}^{3}$ ROI was drawn ventrally in the pons (Fig. 1C) equidistantly from the LC ROIs. The PT ROI was projected to all LC images. 

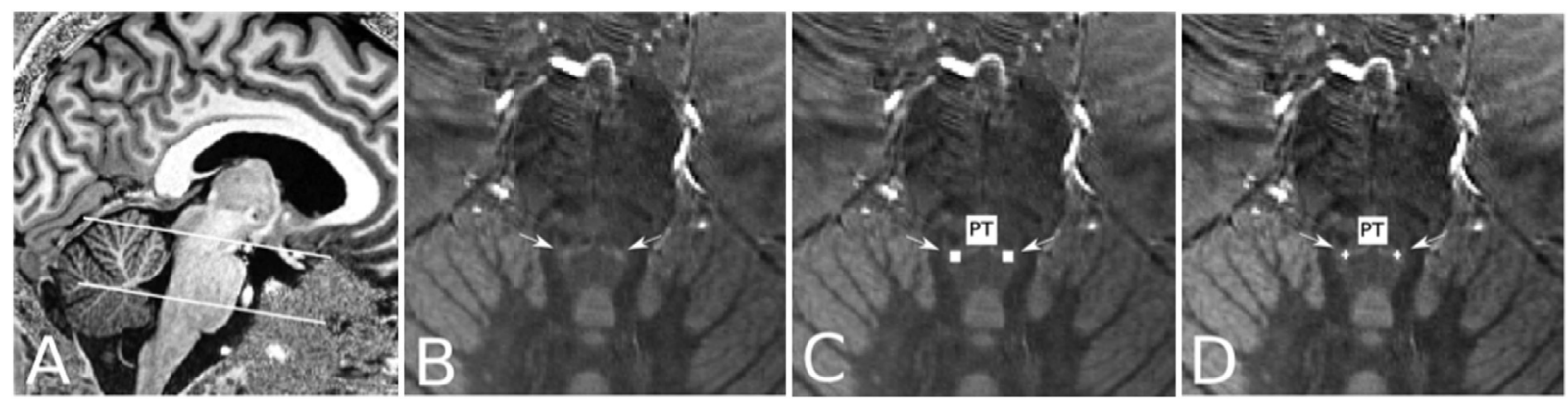

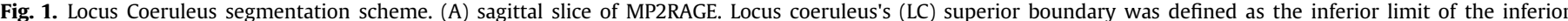

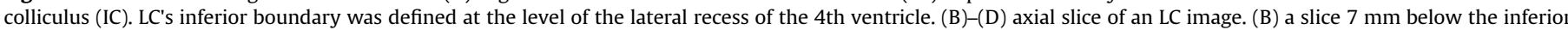

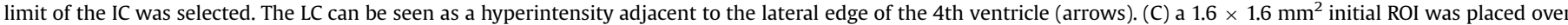

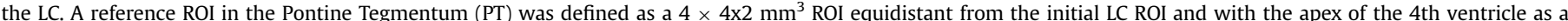
dorsal limit. (D) a $1.2 \times 1.2 \mathrm{~mm}^{2}$ cross, centered over the voxel of the initial LC ROI with the maximum intensity, was defined as the LC ROI.

Table 2

Inter-rater agreement of LC segmentations.

\begin{tabular}{llll}
\hline TSE and MT-TFL images & N & Mean & SD \\
\hline 3T MT-TFL & 5 & 0.71 & 0.13 \\
7T MT-TFL single acquisition & 6 & 0.75 & 0.11 \\
7T MT-TFL 2-average & 5 & 0.91 & 0.09 \\
3T TSE & 6 & 0.86 & 0.14 \\
\hline
\end{tabular}

Note: inter-rater reliability is calculated using the Jaccard ratio.

\subsubsection{Turbo Spin Echo and magnetization transfer turbo flash} comparison

First, we estimated the Line Spread Function (LSF) of MT-TFL and TSE as a measure of resolution. To achieve this, we drew an edge across the SN, since the SN is easier to localize than the LC and still appears hyperintense in MT-weighted images. For each MT-TFL and TSE acquisition, we determined an axial slice at the level of the pontomesencephalic junction. The Edge Response Function (ERF) was determined as a 10-voxel line drawn perpendicular to the SN-related hyperintensity centered along the edge (S-Fig. 4). To ensure that the ERF represented tissue contrast and not noise, the maximum intensity of each ERF was compared to the mean intensity of the image; all ERF maximums were within $70 \%$ of the global mean image intensity. The
LSF, as an approximation of the Point Spread Function (PSF), was calculated by differentiating the ERF. The z-score of the LSF was calculated and an average LSF for each sequence was drawn (S-Fig. 4). In one subject, the SN could not be clearly seen in the 3T acquisitions; the 3T acquisitions of this subject were excluded from the LSF shown.

We then compared the applicability of MT-TFL and TSE for LC imaging at 3T and 7T. First we compared the localization of the LCrelated hyperintensity between 3T TSE, 3T MT-TFL, 7T MT-TFL single acquisition and 7T MT-TFL 2-average (Fig. 2 and S-Fig. 5). The SNR $\left(\mathrm{SNR}=\frac{\mathrm{SI}_{\mathrm{LC}}}{\mathrm{SD}_{\mathrm{LC}}}\right.$, where $\mathrm{SI}_{\mathrm{LC}}$ is the averaged signal intensity for $\mathrm{LC}$ and $\mathrm{SD}_{\mathrm{LC}}$ is the standard deviation of signal intensity for $\mathrm{LC}$ ), as well as $C N R\left(C N R=\frac{S_{L C}-S I_{P T}}{S D_{\text {combined }}}\right.$, where $S_{L C}$ and $S_{I_{P T}}$ are the averaged signal intensity for LC and PT, respectively, and $\mathrm{SD}_{\text {combined }}$ is the standard deviation of the combined LC and PT) were calculated. SNR and CNR per minute were also calculated for 7T MT-TFL single acquisition and 2-average to examine the need for averaging multiple acquisitions with 7T MT-TFL.

To examine the LC border definition, we selected an axial slice at the middle of the $\mathrm{LC}$ in the native space of the $7 \mathrm{~T} \mathrm{~T}_{1}$-weighted acquisition, based on the anatomical description of the LC. We then projected that slice to the space of each scan. A line of 30 voxels was drawn, so that it crossed through both LC ROIs and the
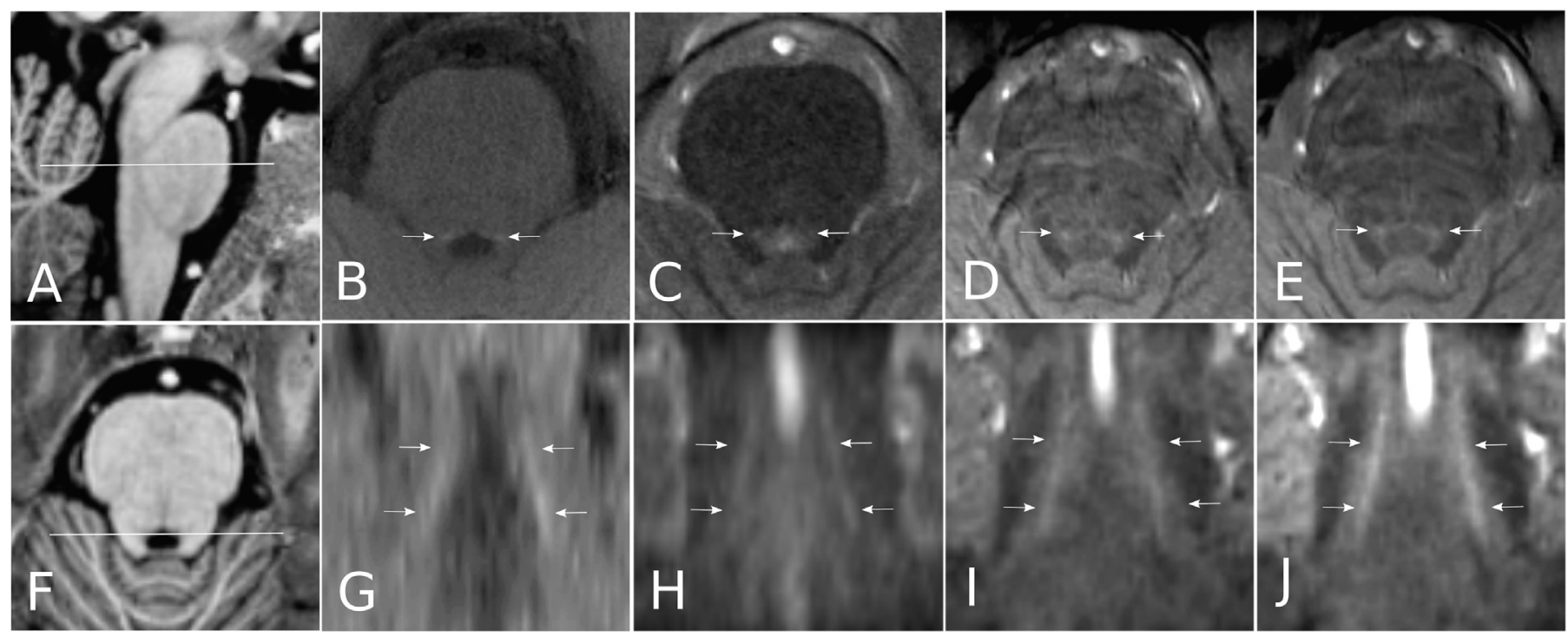

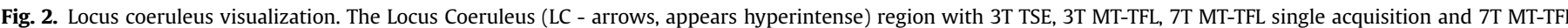

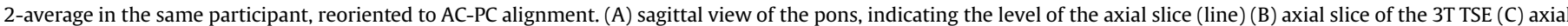

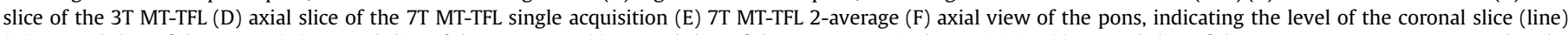

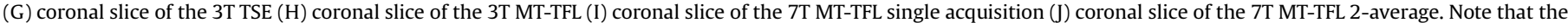
LC hyperintensity is localized at the same position throughout all scans. 

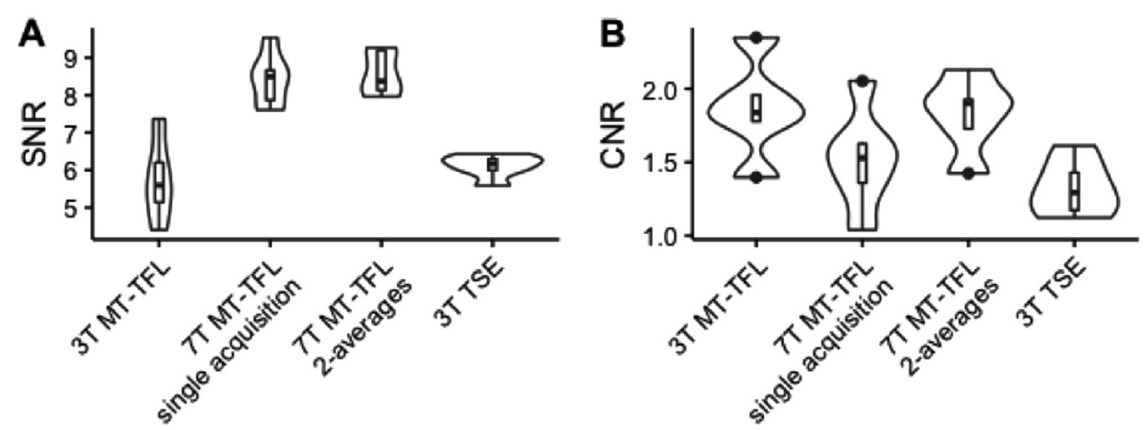

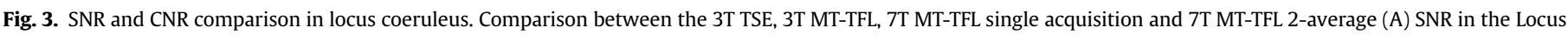
Coeruleus (LC) region. (B) CNR between the LC region and PT (Pontine Tegmentum).

middle of the line was aligned to the apex of the 4th ventricle. The intensity signal was plotted after it was linearly projected to a common scale of $[0,1]$.

Finally, to examine intra- and inter-session reproducibility, we calculated the Jaccard index as described above for the repeated LC volume measurements for the 7T MT-TFL 2-average and 3T TSE.

\subsubsection{Multi-contrast comparison}

In this comparison, we examined whether the LC contrast observed in TSE at 3T is due to MT effects or $\mathrm{R}_{1}$ lengthening, probably due to increased iron concentration, which would also be reflected in increased $\mathrm{R}_{2}{ }^{*}$. The Magnetization Transfer Ratio (MTR) map was calculated on a voxel by voxel basis as MTR $=\frac{\text { MTTFL }- \text { TFL }}{\text { FFL }} \times 100 \%$. An exponential curve was fitted to the magnitude images from the MEGRE using a least squares algorithm to obtain maps of $\mathrm{T}_{2}{ }^{*}$ and $\mathrm{S}_{0}$ (signal at $\mathrm{TE}=0$ ). The $\mathrm{R}_{2}{ }^{*}$ map (i.e. the inverse of $\mathrm{T}_{2}{ }^{*}$ ) was used so that iron-rich areas would appear hyperintense. The $\mathrm{R}_{1}$ map (i.e. the inverse of $T_{1}$ ) was obtained from the MP2RAGE. The MT-TFL, TFL (without the presaturation pulses) and $\mathrm{T}_{1}$ images and MTR, $\mathrm{R}_{2}{ }^{*}$ and $\mathrm{S}_{0}$ maps were visually examined for contrast in the $\mathrm{LC}$ region. The $\mathrm{LC}$ and PT ROIs drawn on the 3T TSE were projected to the MTR and $\mathrm{R}_{2}{ }^{*}$ maps. Based on previous MT-GRE experiments, we expected to observe an increase in MT-TFL signal intensity in LC compared to PT (Chen et al., 2014; Nakane et al., 2008). A possible increase in MT-TFL signal intensity in LC could either relate to MT effects, in which case we would find an increase in MTR, or to an increase in the slightly T2*-weighted TFL on its own. Due to the suggested iron increase in LC (Sasaki et al., 2006), we also hypothesized that we might observe an increase in $\mathrm{R}_{2}{ }^{*}$ and $\mathrm{R}_{1}$ in LC compared to PT. The average intensity across each ROI was calculated and entered into a one-sided Wilcoxon paired signed-rank test for MT-TFL and TFL images and MTR, $\mathrm{R}_{2}{ }^{*}, \mathrm{~S}_{0}$ and $\mathrm{R}_{1}$ maps. Significance was set at $\mathrm{p}<0.05$, False Discovery Rate (FDR) corrected.

\subsubsection{Previously acquired 7 Tesla multi-echo GRE comparison}

To investigate contrast in the LC region in a previously acquired 7T ME-GRE and MP2RAGE dataset for which no individual 3T TSE images were available to extract individual LC ROIs from, we employed the LC template of Keren et al. (2009). The LC template was made from a dataset of 3T TSE images with a similar protocol to our own 3T TSE. The LC region was projected via the diffeomorphic transform from MNI space to each ME-GRE and MP2RAGE with ANTS. Similar to above, a reference ROI was drawn manually in the pontine tegmentum (PT) in the space of the $7 \mathrm{~T}_{1}$-weighted image and projected via BBR to the ME-GRE. The average intensity across each ROI in $\mathrm{R}_{2}{ }^{*}, \mathrm{~S}_{0}$ from the ME-GRE and $R_{1}$ from the MP2RAGE was calculated. Since, similar to above, we expected that we may observe an increase in $R_{2}{ }^{*}$ and $R_{1}$ values in LC compared to PT, due to iron deposition, we employed a one-sided Wilcoxon paired signed-rank test for $\mathrm{R}_{2}{ }^{*}, \mathrm{~S}_{0}$ and $\mathrm{R}_{1}$ maps. Significance was set at $\mathrm{p}<0.05$, FDR corrected.

\section{Results}

\subsection{Locus coeruleus visualization with $3 T$ Turbo Spin Echo and 3 and $7 T$ magnetization transfer turbo flash}

In all participants, the MT-TFL showed contrast in the LC region, close to the 4th ventricle. This was the case for 3T MT-TFL, as well as the 7T MT-TFL acquisitions (Fig. 2, S-Fig. 5). Inter-rater agreement was high across sequences, though it was lowest for 3T MT-TFL (Table 2). The advantage that the higher resolution in the coronal plane of 7T MT-TFL acquisition offers compared to 3T TSE and 3T MT-TFL is evident in Fig. 2G-J. LC contrast showed variation between hemispheres and between participants in size, as previously reported (Keren et al., 2009). Importantly though, the position of the LC hyperintensity was consistent across the 3T TSE, 3T MT-TFL, 7T MT-TFL single acquisition and 7T MT-TFL 2-average images (S-Fig. 5).

To compare the resolving power of the MT-TFL and TSE acquisitions, the LSF across the edge of SN and surrounding tissue was determined, since SN is the largest NM-rich nucleus in the brain. MT-TFL acquisitions showed a sharper incline compared to the 3T TSE, thus validating that high in-plane resolving power is retained with MT-TFL sequences (S-Fig. 4).

To validate the image quality in the LC for the MT-TFL acquisitions, the mean SNR and CNR of the LC were estimated for 3T TSE, 3T MTTFL, 7T MT-TFL single acquisition and 7T MT-TFL 2-average (Table 3). 7T MT-TFL images had the highest mean SNR and comparable CNR, even though they were acquired at a resolution 4-times higher than the 3T TSE and 2-times higher than the 3T MT-TFL. Note that the 7T MT-TFL does not appear to benefit from multiple averages; this is particularly clear when SNR and CNR efficiency are considered: mean SNR and CNR per minute were higher for 7T MT-TFL single acquisition $(\mathrm{M}=1.82, \mathrm{SD}=0.15$ and $\mathrm{M}=0.33, \mathrm{SD}=0.07$ respectively) compared to 7T MT-TFL 2-average $(\mathrm{M}=0.93, \mathrm{SD}=0.007$ and $\mathrm{M}=0.2$, $\mathrm{SD}=0.03)$. This is partially expected since (1) we acquired highresolution images at a brain area prone to motion (2) the 3D TFL readout might result in increase motion susceptibility, despite its evident resolution advantages. 7T MT-TFL single acquisition in our study reliably allowed localization of the LC with high SNR and CNR; this is promising as a fast high-resolution LC imaging technique. 3T MT-TFL's SNR was similar to the SNR obtained from 3T TSE.

To emphasize the advantage of the increased SNR at 7T, as well as the MT-TFL acquisition, in LC segmentation, we examined the linearly

Table 3

SNR and CNR values in the LC region across images.

\begin{tabular}{llll}
\hline TSE and MT-TFL images & N & Mean SNR (SD) & Mean CNR (SD) \\
\hline 3T MT-TFL & 5 & $5.74(1.12)$ & $1.86(0.34)$ \\
7T MT-TFL single acquisition & 6 & $8.42(0.71)$ & $1.52(0.34)$ \\
7T MT-TFL 2-average & 5 & $8.59(0.61)$ & $1.82(0.26)$ \\
3T TSE & 6 & $6.10(0.30)$ & $1.32(0.19)$ \\
\hline
\end{tabular}



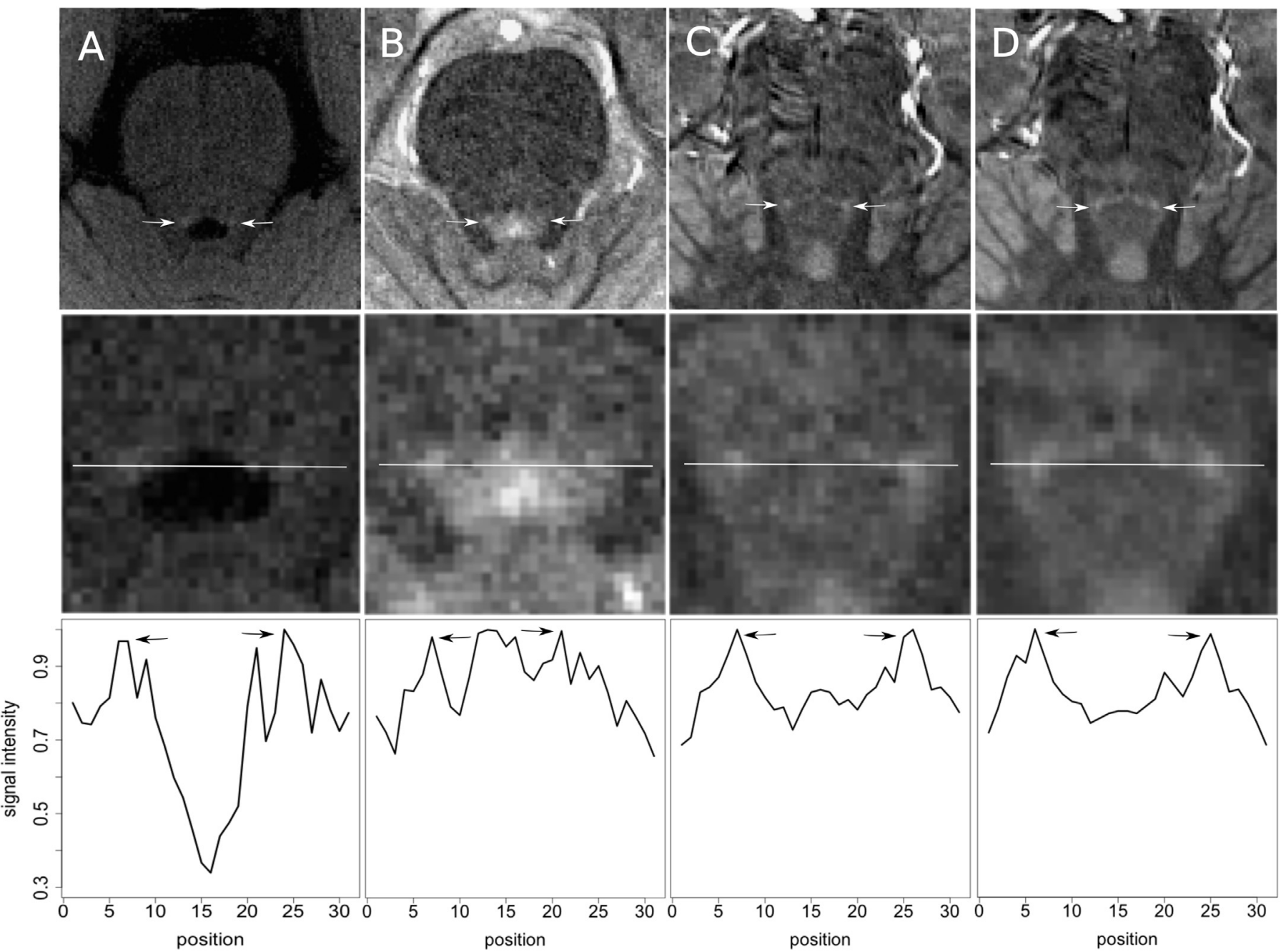

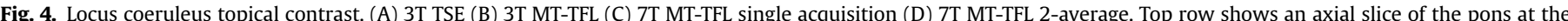

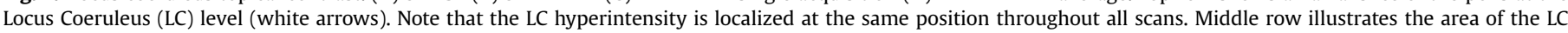

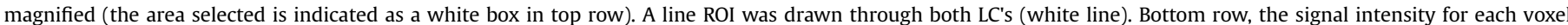

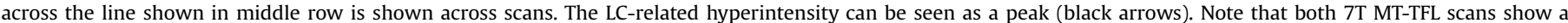
smoother and sharper increase in intensity in the LC region, in accordance with the higher SNR and CNR estimates. This may facilitate LC segmentation.

scaled intensity through an LC slice (Fig. 4, S-Fig. 6). The signal intensity observed was less noisy, reflecting the SNR gain at 7T (Table 3). This allowed for the LC-related intensity peak to be made out clearer in both 7T MT-TFL images compared to 3T images, which may help LC segmentation. Note that the CSF in 4th ventricle does not get suppressed in the MT-TFL acquisitions; CSF has a low macromolecular content and is therefore minimally affected by the MT saturation. This results in a peak in the middle of the intensity line in MT-TFL acquisitions compared to the TSE. This does not directly affect the LC segmentation, since the 4th ventricle limits are clearly defined and partial volume effects are limited by the high-resolution.

Finally, we examined the reproducibility of the LC segmentation in one participant for 7T MT-TFL 2-average and 3T TSE by calculating the Jaccard ratio. 3T TSE and 7T MT-TFL 2-average showed similar intra-session reproducibility in the participant we tested multiple times (7T MT-TFL 2-average Jaccard ratio $=0.61$, 3T TSE Jaccard ratio $=0.67$ ) and inter-session reproducibility (7T MT-TFL 2-average Jaccard ratio $=0.67,3 \mathrm{~T}$ TSE Jaccard ratio $=0.68$ ).

\subsection{Multi-contrast comparison}

After demonstrating the utility of MT-weighted images for LC imaging at 3T and 7T, we explored whether the LC contrast observed in the 3T TSE predominantly relates to MT effects or whether another mechanism, such as $R_{1}$ and $R_{2}{ }^{*}$ increase is at play. To achieve this, we projected the LC and PT segmentations from the 3T TSE to the MT-TFL, TFL (without saturation pulses) and MTR, $\mathrm{R}_{2}{ }^{*}, \mathrm{~S}_{0}$ and $\mathrm{R}_{1}$ maps and we calculated the mean intensity of the LC and PT (Fig. 5). While we did not detect higher intensity in LC using MT-TFL (Mdn $=116.97$, $\mathrm{IQR}=11.61)$ than in PT ( $\mathrm{Mdn}=112.66, \mathrm{IQR}=5.73)$, we detected a possible trend in that direction, $\mathrm{Z}=21.00, \mathrm{p}_{\text {corrected }}=0.09$ ( puncorrected $=0.02$ ). Similarly in the MTR map we detected a possible trend for higher LC intensity ( $\mathrm{Mdn}=-21.93$, IQR $=6.24$ ) compared to the PT intensity ( $\mathrm{Mdn}=-23.08, \mathrm{IQR}=4.10$ ), $\mathrm{Z}=20.00$, $p_{\text {corrected }}=0.09$ ( $\mathrm{p}_{\text {uncorrected }}=0.03$ ). The LC intensities in $\mathrm{R}_{2}{ }^{*}, \mathrm{~S}_{0}, \mathrm{R}_{1}$ and TFL (without presaturation pulses) maps were not significantly different from the PT intensity (all $p>0.05$ ).

Careful visual inspection confirmed that an LC intensity increase, such as the one detected in TSE or MT, was not evident in $\mathrm{R}_{2}{ }^{*}, \mathrm{~S}_{0}$ and $\mathrm{R}_{1}$. We observed instead a potential decrease in $\mathrm{R}_{2}{ }^{*}$ due to the (minimal) grey and white matter contrast between LC and PT, but it was not specific to the LC compared to the rest of the grey matter surrounding the 4 th ventricle. We should note that a hyperintensity was also observed in the $R_{2}{ }^{*}$ map close to the $\mathrm{LC}$ region (Fig. $6 \mathrm{E}$ ); however, anatomically it did not fit the LC and further examination of the neighboring slices revealed it to be most likely a vein. The LC 

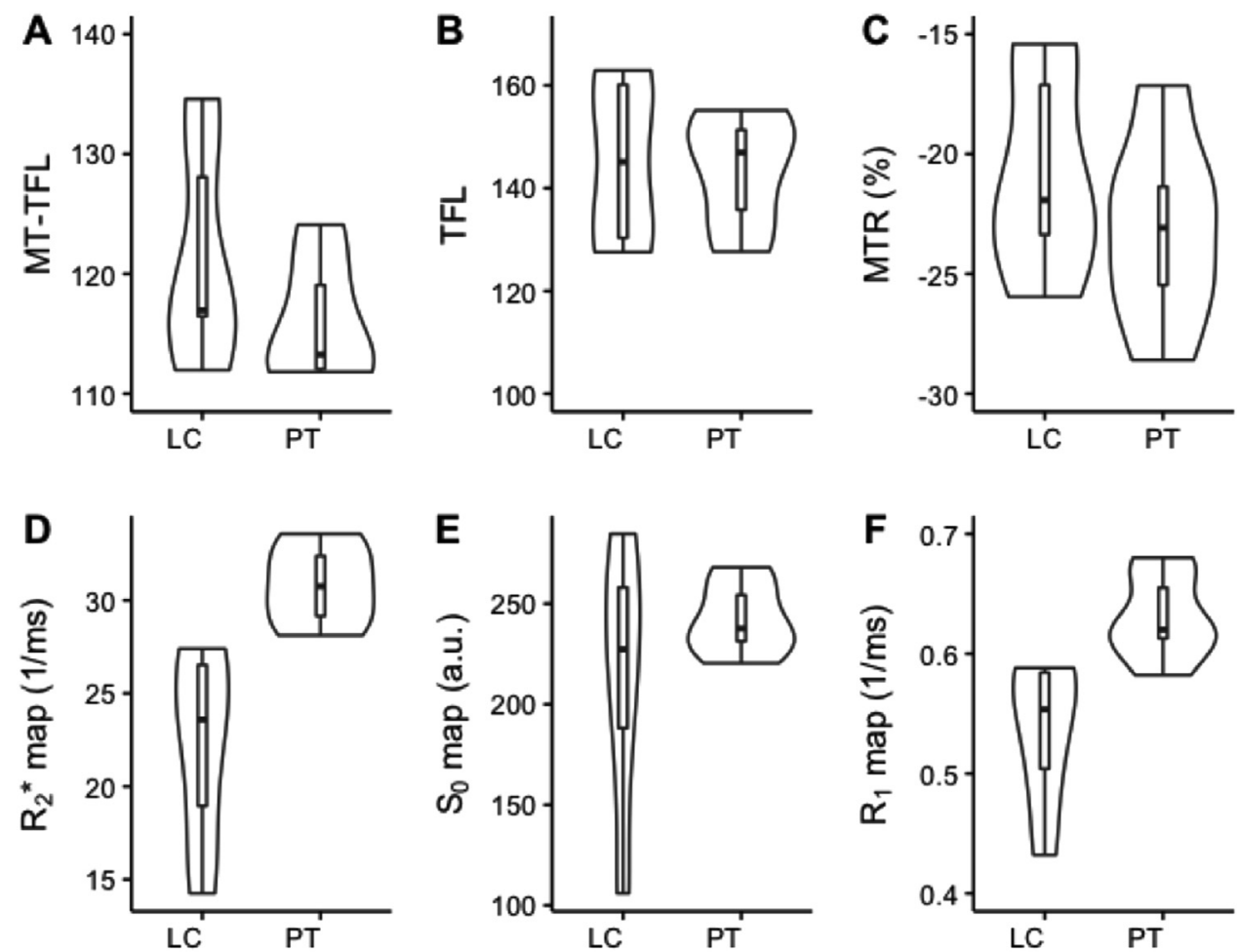

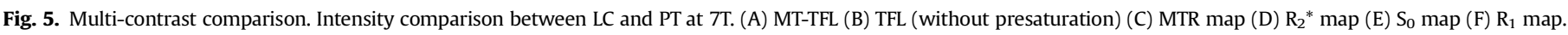

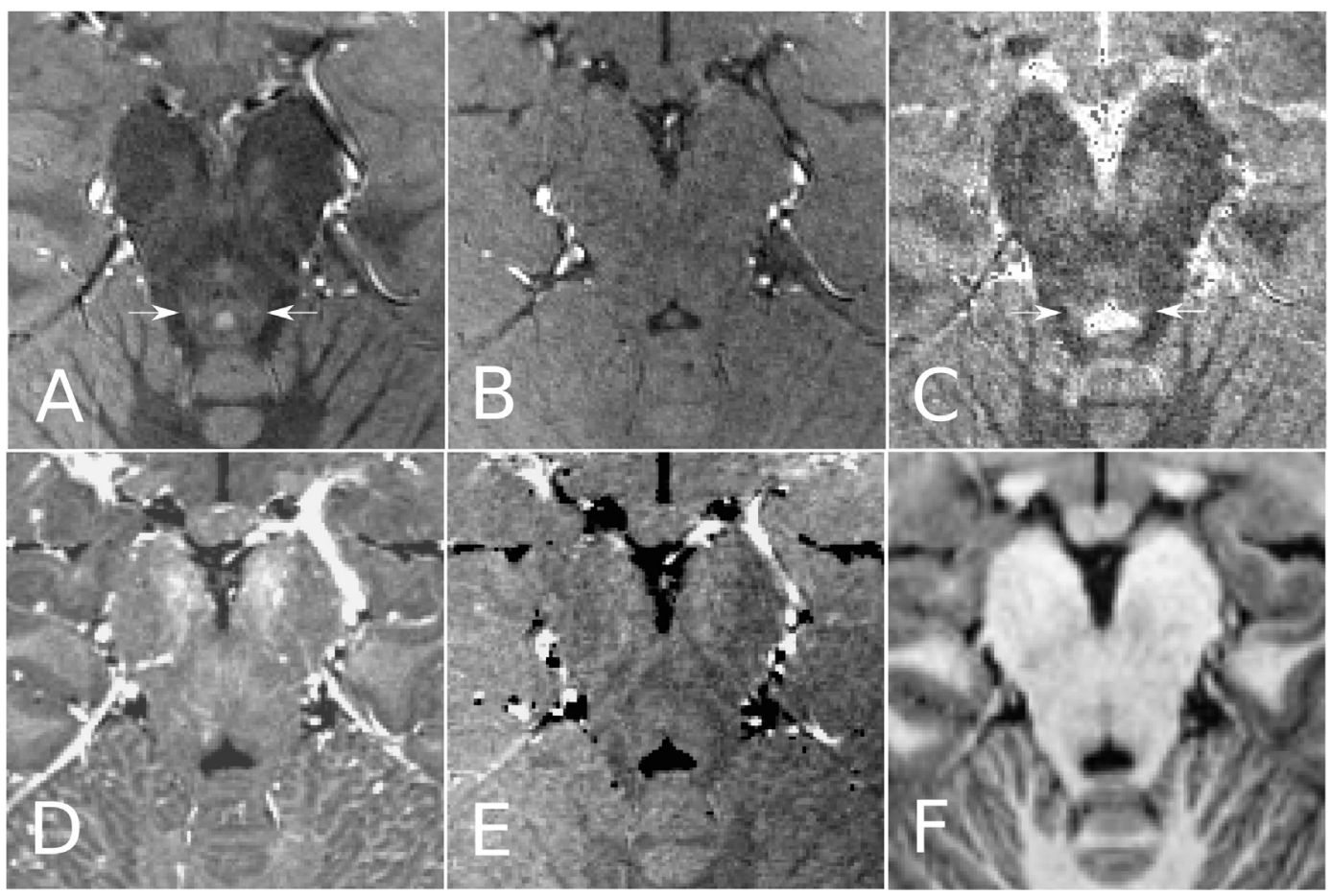

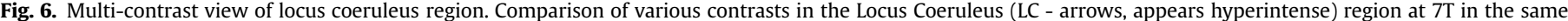

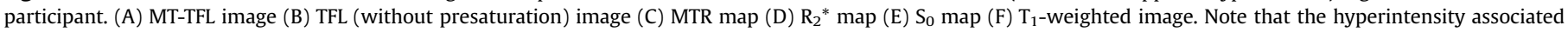
with the LC is visible in MT-TFL and MTR images, but not in the $\mathrm{R}_{2}{ }^{*}$.

was visible in some slices in the MTR (Fig. 6D), but was judged too noisy for segmentation of such a small structure.

\section{3. $R_{2}{ }^{*}$ and $S_{0}$ comparison on the previously acquired 7T ME-GRE} dataset

Since $R_{2}{ }^{*}$ and $R_{1}$ increase have previously been suggested as a possible mechanism for LC imaging (Sasaki et al., 2006; Silvy Collin et al., 2014), we decided to examine a previously acquired ME-GRE and MP2RAGE dataset including the LC region to validate our results (Fig. 7). Again, the mean LC intensity in the $\mathrm{R}_{2}{ }^{*}$ and $\mathrm{R}_{1}$ maps was not significantly different than the PT intensity $(p>0.05)$. However, the LC intensity in $\mathrm{S}_{0}(\mathrm{Mdn}=315.69, \mathrm{IQR}=52.30)$ was significantly greater than the PT intensity in $\mathrm{S}_{0}(\mathrm{Mdn}=295.54$, 

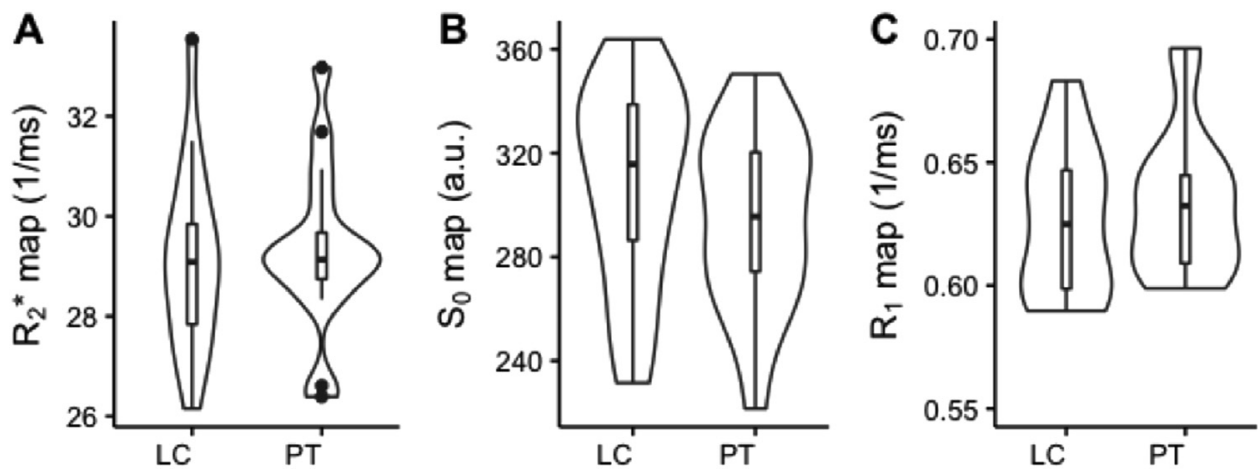

Fig. 7. Intensity comparison between the LC and the PT in the previously acquired $7 T$ multi echo gradient echo dataset. (A) $R_{2}{ }^{*}$ map (B) $S_{0}$ map (C) $R_{1}$ map.

$\mathrm{IQR}=45.81), \mathrm{Z}=122, p_{\text {corrected }}=0.006$ ( $\mathrm{p}_{\text {uncorrected }}=0.002$ ). Visual inspection confirmed that LC contrast was not evident in the $\mathrm{R}_{2}{ }^{*}$ or $\mathrm{R}_{1}$ map. Visual check of the $\mathrm{S}_{0}$ image showed that the contrast difference between LC and PT was due to the (minimal) grey and white matter contrast between them, but was not specific to the LC compared to the rest of the grey matter surrounding the 4 th ventricle.

To conclude, we did not observe an $\mathrm{R}_{2}{ }^{*}$ or $\mathrm{R}_{1}$ increase in the LC region compared to a control ROI. In MT, instead, we did observe a hyperintensity in the $\mathrm{LC}$ region.

\section{Discussion}

In this study, we developed for the first time a MT-weighted sequence for LC imaging at 7T and translated this successfully back to application at 3T. We succeeded in accurately imaging the LC at a higher spatial resolution, while reducing the acquisition time (TA) compared to the current spin-echo based "gold standard" and retaining high SNR and CNR. Furthermore, we investigated the source of the image contrast in the LC and found it to be predominantly related to MT effects.

LC imaging is of great scientific interest for cognitive neuroscience and for several neuropsychiatric disorders. Previous pharmacological studies have shown that the LC modulates attention, memory and the response of the sympathetic system (Berridge and Waterhouse, 2003; Samuels and Szabadi, 2008; Sara, 2009; Sterpenich et al., 2006). Furthermore, from a clinical perspective, the LC has been suggested to be the first region in the brain that shows early tau pathology in preclinical AD (Braak and Del Tredici, 2011) and the LC atrophies in a variety of other diseases including PTSD, PD and depression (Hendrickson and Raskind, 2016; Weiss et al., 1994). Relatively few in vivo structural and functional MRI studies have explored its function and dysfunction at 3T (Clewett et al., 2016; Jacobs et al., 2015; Mather et al., 2015; Murphy et al., 2014) and none at 7T as far as we are aware, reflecting the challenges to accurately localize the structure. By employing MT-weighted images, we have shown now that LC imaging can be done accurately and fast at 7T, opening up many new opportunities.

Producing MT-weighted images in vivo is challenging at 7T. While MT-weighted images are usually obtained by interleaving a saturation pulse with each line of k-space readout, this approach is both time-consuming and restricted at 7T, due to the SAR increase. Instead, we employed a train of saturation pulses followed by a 3DTFL readout (Mougin et al., 2010), which achieved sufficient MT saturation for LC imaging at 7T. In comparison to the 3T MT-TFL acquisition, we observed high SNR and similar CNR at 7T. We should note that in our experiment we focused on optimizing LC visualization for each field strength within a similar TA (12 min) and inplane resolution. We therefore took advantage of the high SNR of
MT-TFL in LC at 7T to double the through-plane resolution compared to 3T. Increased resolution is critical to reduce partial volume effects in a structure that is both small and close to 4th ventricle. The CNR was similar between the 3T and 7T (Fig. 3). In our experiment, we opted to set the amplitude of the saturation pulses at the maximum allowed for a $55 \mathrm{~kg}$ participant for each field strength, to maximize MT weighting, which we hypothesized provides LC contrast. The SAR increase at 7T forced us to employ a train of saturation pulses of half the amplitude compared to 3T. This results in reduced MT weighting during recovery, as shown in Mougin et al. (2013), which may explain the reduced CNR gain at 7T compared to SNR. We should note though that the resolution at 7T was twice the one at 3T; assuming that CNR and SNR is proportional to voxel volume, this would translate into higher SNR and CNR values at 7T for the same spatial resolution as used in 3T. Overall, the increased resolution and SNR of MT-TFL at 7T allowed for a more reliable localization of the LC compared to 3T; this is reflected in the higher inter-rater agreement (Table 2). Finally, we observed that LC localization was feasible at 7T also with a single acquisition ( $\mathrm{TA}=4: 30 \mathrm{~min}$ ) while still retaining high SNR and CNR, which is promising for clinical applications where short scanning times are important for patient comfort and to avoid image corruption by motion (particularly relevant for brainstem imaging). 7T MRI is becoming increasingly available in the clinical setting, which is promising for the exploration of function of small brainstem nuclei, such as the LC, in disease.

The LC localization was consistent between 3T TSE and 3 and 7T MT-TFL acquisitions in our results. The TSE, as a spin-echo-based sequence, has relatively long TA and low SNR and its application at $7 \mathrm{~T}$ is challenging due to the multiple RF pulses it employs. Instead, gradient-echo approaches use less RF power, which allowed us to increase MT-weighting to facilitate LC imaging at 7T. Furthermore, compared to the 2D TSE approach, the MT-TFL is a GRE-based 3D sequence, which affords increased coverage/reduced TA, while the MT saturation is not incidental and can be set at an optimal value. LC contrast was shown in our pilot (S-Fig. 2), as well as in the literature (Trujillo et al., 2015), to be sensitive to frequency offset manipulation. This should be explored further in future studies. MT-TFL may allow for more accurate LC segmentation (Fig. 4), though this was specific to 7T. Our MT-TFL implementation achieved two-times higher resolution at 3T and four-times higher resolution at 7T with similar SNR and CNR compared to the 3T TSE.

The source of the LC MRI contrast in $\mathrm{T}_{1}$-weighted TSE is under debate: since the main function of NM in LC is thought to be that of an iron chelator (Enochs et al., 1994), it was speculated that iron binding with $\mathrm{NM}$ macromolecules might result in $\mathrm{R}_{1}$ and $\mathrm{R}_{2}{ }^{*}$ increase in the LC (Sasaki et al., 2006). Another possibility is that the short TE in TSE accentuates incidental MT effects. We investigated this question with a within-participant design, which is necessary for a structure with a relatively high anatomical variance (Keren et al., 2015). In our results, we were not able to detect a $R_{1}$ or $R_{2}{ }^{*}$ 
increase in the LC region. The lack of $\mathrm{R}_{2}{ }^{*}$ contrast in LC was partially unexpected: an $\mathrm{R}_{2}{ }^{*}$ increase in the similarly NM-rich $\mathrm{SN}$ is well documented (Lee et al., 2016) though we should note that NM and iron are not necessarily evenly distributed across pars compacta and pars reticulata (Langley and colleagues have suggested that the two compartments can be differentiated based on a $\mathrm{T}_{2}{ }^{*}$ and MT images comparison) (Langley et al., 2015). Histological studies have indicated though, that the LC shows relatively low iron deposition regardless of age in comparison to SN (Zecca et al., 2004). Furthermore, NM on its own is known to be lowly paramagnetic (Ju et al, 2013; Lee et al., 2016), but this effect is detectable at high concentrations only (Trujillo et al., 2016). In our results, MT-TFL and MTR images showed contrast in the LC region that matched that of the 3T TSE. Furthermore, we did not detect LC contrast in the TFL sequence without saturation $\left(\mathrm{T}_{2}{ }^{*}\right.$-weighted). We conclude therefore that LC contrast seems to relate predominantly to MT-effects.

MRI contrast in the LC region is thought to reflect NM concentration: the contrast is specific to the NM-accumulating regions, like the LC and SN (Sasaki et al., 2006), and not other catecholaminergic structures, such as the raphe nucleus (Keren et al., 2015), the ventral tegmental or retrorubal areas (Lee et al., 2016). Furthermore, MRI contrast has been shown to increase with increasing concentration of NM in both LC (Keren et al., 2015) and SN (Kitao et al., 2013). Therefore, MT imaging in the LC region might provide a marker for NM concentration.

NM accumulates with aging (Zecca et al., 2004), but its concentration in the SN is reduced in PD (Zecca et al., 2002), and in LC in AD (Hoogendijk et al., 1995; Iversen et al., 1983). A similar finding of reduced TSE contrast has been reported in vivo with MRI in both PD and AD (Castellanos et al., 2015; Schwarz et al., 2016; Takahashi et al., 2015). Since NM concentration might reflect the loss or dysfunction of dopaminergic and noradrenergic neurons respectively, an in vivo marker of NM concentration is of great clinical importance. MT effects have been used as a marker for a variety of diseases (Anik et al., 2007; Karampekios et al., 2005; Ridha et al., 2007) and may be promising as a potential marker for NM. The LC showed high signal intensity compared to adjacent areas in MTweighted images (Fig. 5), which would indicate that the LC is less susceptible or recovers faster from saturation. The precise mechanism of that is unclear: NM does not reach concentrations high enough to significantly affect bound-to-free pool size ratio (Trujillo et al., 2016). One may speculate that the contrast observed is due to the effect of a short $T_{1}$ in the bound pool, as pointed out by (Langley et al., 2015) or due to the influence of $T_{1}$ and $T_{2}$ on the magnetization transfer spectra of the LC compared to adjacent grey matter (Trujillo et al., 2016). A quantitative MT experiment may shed light on the precise contrast mechanism (Henkelman et al., 1993).

There are several limitations in this study. First, our main purpose was to establish a high-resolution sequence for LC imaging in UHF and therefore our first cohort had a relatively small sample size $(\mathrm{N}=6)$, while in our independent cohort $(\mathrm{N}=18)$, no MT-weighted images were available to match with our $T_{2}{ }^{*}$ and $T_{1}$ maps. For the precise MT contrast mechanism in the LC to be untangled, a study including $T_{1}$ and $T_{2}$ and several MT measurements would be needed. Second, the reproducibility of LC segmentation with the MT-TFL, particularly in a clinical setting, needs to be appraised, given the sensitivity of 3D acquisitions to motion. Third, our study was based on young, healthy participants who potentially have lower levels of iron (Daugherty and Raz, 2015) and NM (Zecca et al., 2002) accumulated in the brain compared to older individuals. Fourth, while the LC did not show any visible contrast in $\mathrm{R}_{2}{ }^{*}$ or $\mathrm{R}_{1}$ compared to its adjacent areas, we observed lower $\mathrm{R}_{2}{ }^{*}$ and $\mathrm{R}_{1}$ in LC compared to PT in our first cohort (Fig. 5D,F), which likely relates to partial volume effects. Finally, in our signal intensity comparisons we employed an adjacent grey matter control area in the pons (PT). This is the method commonly used in the LC literature (Clewett et al., 2016; Mather et al,
2017; Ohtsuka et al., 2013; Takahashi et al., 2015) and therefore may be most informative to researchers in the field. A PT ROI though may have its own unique tissue properties or may be differentially affected in aging or disease, as noted in (Keren et al., 2009). One advantage of a quantitative MT method compared to TSE is that it provides a metric independent of a control area.

\subsection{Conclusion}

In this study, we showed for the first time the feasibility of imaging the LC in vivo at 7T by employing a novel, fast, high-resolution, MT-based sequence. We furthermore investigated the source of LC contrast and showed that it relates to MT effects.

\section{Acknowledgements}

We would like to thank Linda Pagen, MSc and Dr. Wilma van de Berg (VUmc, anatomy and neurosciences, Amsterdam, the Netherlands) for the help they provided with the locus coeruleus demarcations and neuroanatomical guidance.

We would like to thank the University Fund Limburg/Brains Unlimited Pioneering Fund for funding support (SWOL/ 5.2015.1.07). HILJ receives support from the Netherlands Organisation for Scientific Research (VENI/016.158.084). Kamil Uludag and Dimo Ivanov are funded by the Netherlands Organization for Scientific Research (VIDI/452.11.002) (PI Kamil Uludag). Benedikt Poser is partially funded by a National Institue for Health grant (R01MH/111444) (PI David Feinberg).

\section{Appendix A. Supplementary data}

Supplementary data related to this article can be found at http://dx.doi.org/10.1016/j.neuroimage.2017.07.045.

\section{References}

Anik, Y., Iseri, P., Demirci, A., Komsuoglu, S., Inan, N., 2007. Magnetization transfer ratio in early period of Parkinson disease. Acad. Radiol. 14, 189-192.

Aston-Jones, G., Rajkowski, J., Cohen, J., 2000. Locus coeruleus and regulation of behavioral flexibility and attention. Prog. Brain Res. 126, 165-182.

Avants, B.B., Tustison, N.J., Song, G., Cook, P.A., Klein, A., Gee, J.C., 2011. A reproducible evaluation of ANTs similarity metric performance in brain image registration. Neurolmage 54, 2033-2044.

Baker, K.G., Tork, I., Hornung, J.P., Halasz, P., 1989. The human locus coeruleus complex: an immunohistochemical and three dimensional reconstruction study. Exp. Brain Res. 77, 257-270.

Berridge, C.W., Waterhouse, B.D., 2003. The locus coeruleus-noradrenergic system: modulation of behavioral state and state-dependent cognitive processes. Brain Res. Brain Res, Rev, 42, 33-84.

Braak, H., Del Tredici, K., 2011. Alzheimer's pathogenesis: is there neuron-to-neuron propagation? Acta Neuropathol. 121, 589-595.

Busch, C. Bohl, J., Ohm, T.G., 1997. Spatial, temporal and numeric analysis of Alzheimer changes in the nucleus coeruleus. Neurobiol. Aging 18, 401-406.

Castellanos, G., Fernandez-Seara, M.A., Lorenzo-Betancor, O., Ortega-Cubero, S., Puigvert, M., Uranga, J., Vidorreta, M., Irigoyen, J., Lorenzo, E., Munoz-Barrutia, A., Ortiz-de-Solorzano, C., Pastor, P., Pastor, M.A., 2015. Automated neuromelanin imaging as a diagnostic biomarker for Parkinson's disease. Mov. Disord. 30, 945-952.

Chen, X., Huddleston, D.E., Langley, J., Ahn, S., Barnum, C.J., Factor, S.A., Levey, A.I., $\mathrm{Hu}, \mathrm{X}$., 2014. Simultaneous imaging of locus coeruleus and substantia nigra with a quantitative neuromelanin MRI approach. Magn. Reson Imaging 32 . 1301-1306.

Clewett, D.V., Lee, T.-H., Greening, S., Ponzio, A., Margalit, E., Mather, M., 2016. Neuromelanin marks the spot: identifying a locus coeruleus biomarker of cognitive reserve in healthy aging. Neurobiol. Aging 37, 117-126.

Daugherty, A.M., Raz, N., 2015. Appraising the role of iron in brain aging and cognition: promises and limitations of MRI. Methods. Neuropsychol. Rev. 25, 272-287.

Dixon, W.T. Engels, H., Castillo, M., Sardashti, M., 1990. Incidental magnetization transfer contrast in standard multislice imaging. Magn. Reson Imaging 8 , 
$417-422$.

Enochs, W.S., Sarna, T., Zecca, L., Riley, P.A., Swartz, H.M., 1994. The roles of neuromelanin, binding of metal ions, and oxidative cytotoxicity in the pathogenesis of Parkinson's disease: a hypothesis. J. Neural Transm. Park Dis. Dement. Sect. 7, 83-100.

Fernandes, P., Regala, J., Correia, F., Gonçalves-Ferreira, A.J., 2012. The human locus coeruleus 3-D stereotactic anatomy. Surg. Radiologic Anat. 34, 879-885.

German, D.C., Walker, B.S., Manaye, K., Smith, W.K., Woodward, D.J., North, A.J. 1988. The human locus coeruleus: computer reconstruction of cellular distribution. J. Neurosci. 8, 1776-1788.

Haacke, E.M., Cheng, N.Y., House, M.J., Liu, Q., Neelavalli, J., Ogg, R.J., Khan, A., Ayaz, M., Kirsch, W., Obenaus, A., 2005. Imaging iron stores in the brain using magnetic resonance imaging. Magn. Reson Imaging 23, 1-25.

Hamilton, M., 1960. A rating scale for depression. J. Neurol. Neurosurg. Psychiatry 23, 56-62.

Hendrickson, R.C., Raskind, M.A., 2016. Noradrenergic dysregulation in the pathophysiology of PTSD. Exp. Neurol. 284, 181-195.

Henkelman, R.M., Huang, X., Xiang, Q.S., Stanisz, G.J., Swanson, S.D., Bronskill, M.J., 1993. Quantitative interpretation of magnetization transfer. Magn. Reson Med. 29, 759-766.

Hennig, J., Nauerth, A., Friedburg, H., 1986. RARE imaging: a fast imaging method for clinical MR. Magn. Reson Med. 3, 823-833.

Hoogendijk, W.J., Pool, C.W., Troost, D., van Zwieten, E., Swaab, D.F., 1995. Image analyser-assisted morphometry of the locus coeruleus in Alzheimer's disease, Parkinson's disease and amyotrophic lateral sclerosis. Brain 118 (Pt 1), 131-143.

Iglesias, J.E., Van Leemput, K., Bhatt, P., Casillas, C., Dutt, S., Schuff, N., Truran-Sacrey, D., Boxer, A., Fischl, B., 2015. Bayesian segmentation of brainstem structures in MRI. NeuroImage 113, 184-195.

Iversen, L.L., Rossor, M.N., Reynolds, G.P., Hills, R., Roth, M., Mountjoy, C.Q., Foote, S. L., Morrison, J.H., Bloom, F.E., 1983. Loss of pigmented dopamine-beta-hydroxylase positive cells from locus coeruleus in senile dementia of Alzheimer's type. Neurosci. Lett. 39, 95-100.

Jacobs, H.I., Wiese, S., van de Ven, V., Gronenschild, E.H., Verhey, F.R., Matthews, P. M., 2015. Relevance of parahippocampal-locus coeruleus connectivity to memory in early dementia. Neurobiol. Aging 36, 618-626.

Ju, K.-Y., Lee, J.W., Im, G.H., Lee, S., Pyo, J., Park, S.B., Lee, J.H., Lee, J.-K., 2013. Bioinspired, melanin-like nanoparticles as a highly efficient contrast agent for T1weighted magnetic resonance imaging. Biomacromolecules 14, 3491-3497.

Karampekios, S., Papanikolaou, N., Papadaki, E., Maris, T., Uffman, K., Spilioti, M., Plaitakis, A., Gourtsoyiannis, N., 2005. Quantification of magnetization transfer rate and native $\mathrm{T} 1$ relaxation time of the brain: correlation with magnetization transfer ratio measurements in patients with multiple sclerosis. Neuroradiology 47, 189-196.

Keren, N.I., Lozar, C.T., Harris, K.C., Morgan, P.S., Eckert, M.A., 2009. In vivo mapping of the human locus coeruleus. NeuroImage 47, 1261-1267.

Keren, N.I., Taheri, S., Vazey, E.M., Morgan, P.S., Granholm, A.-C.E., Aston-Jones, G.S., Eckert, M.A., 2015. Histologic validation of locus coeruleus MRI contrast in postmortem tissue. NeuroImage 113, 235-245.

Kitao, S., Matsusue, E., Fujii, S., Miyoshi, F., Kaminou, T., Kato, S., Ito, H., Ogawa, T., 2013. Correlation between pathology and neuromelanin MR imaging in Parkinson's disease and dementia with Lewy bodies. Neuroradiology 55, 947-953.

Klein, A., Andersson, J., Ardekani, B.A., Ashburner, J., Avants, B., Chiang, M.C., Christensen, G.E., Collins, D.L., Gee, J., Hellier, P., Song, J.H., Jenkinson, M., Lepage, C., Rueckert, D., Thompson, P., Vercauteren, T., Woods, R.P., Mann, J.J., Parsey, R.V., 2009. Evaluation of 14 nonlinear deformation algorithms applied to human brain MRI registration. NeuroImage 46, 786-802.

Klimek, V., Stockmeier, C., Overholser, J., Meltzer, H.Y., Kalka, S., Dilley, G., Ordway, G.A., 1997. Reduced levels of norepinephrine transporters in the locus coeruleus in major depression. J. Neurosci. 17, 8451-8458.

Langkammer, C., Krebs, N., Goessler, W., Scheurer, E., Ebner, F., Yen, K., Fazekas, F., Ropele, S., 2010. Quantitative MR imaging of brain iron: a postmortem validation study. Radiology 257, 455-462.

Langley, J., Huddleston, D.E., Chen, X., Sedlacik, J., Zachariah, N., Hu, X., 2015. A multicontrast approach for comprehensive imaging of substantia nigra. NeuroImage 112, 7-13.

Lee, J.H., Baek, S.Y., Song, Y., Lim, S., Lee, H., Nguyen, M.P., Kim, E.J., Huh, G.Y., Chun, S.Y., Cho, H., 2016. The neuromelanin-related T2* contrast in postmortem human substantia nigra with 7T MRI. Sci. Rep. 6, 32647.

Liu, C., Li, W., Tong, K.A., Yeom, K.W., Kuzminski, S., 2015. Susceptibility-weighted imaging and quantitative susceptibility mapping in the brain. Journal of magnetic resonance imaging. JMRI 42, 23-41.

Marcyniuk, B., Mann, D.M., Yates, P.O., 1986. The topography of cell loss from locus caeruleus in Alzheimer's disease. J. Neurol. Sci. 76, 335-345.

Marques, J.P., Kober, T., Krueger, G., van der Zwaag, W., Van de Moortele, P.F., Gruetter, R., 2010. MP2RAGE, a self bias-field corrected sequence for improved segmentation and T1-mapping at high field. NeuroImage 49, 1271-1281.

Mather, M., Clewett, D., Sakaki, M., Harley, C.W., 2015. Norepinephrine ignites local hot spots of neuronal excitation: how arousal amplifies selectivity in perception and memory. Behav. Brain Sci., 1-100.

Mather, M., Joo Yoo, H., Clewett, D.V., Lee, T.H., Greening, S.G., Ponzio, A., Min, J., Thayer, J.F., 2017. Higher locus coeruleus MRI contrast is associated with lower parasympathetic influence over heart rate variability. NeuroImage 150 , 329-335.

Mougin, O., Clemence, M., Peters, A., Pitiot, A., Gowland, P., 2013. High-resolution imaging of magnetisation transfer and nuclear Overhauser effect in the human visual cortex at 7 T. NMR Biomed. 26, 1508-1517.

Mougin, O.E., Coxon, R.C., Pitiot, A., Gowland, P.A., 2010. Magnetization transfer phenomenon in the human brain at 7 T. Neurolmage 49, 272-281.

Murphy, P.R., O'Connell, R.G., O'Sullivan, M., Robertson, I.H., Balsters, J.H., 2014. Pupil diameter covaries with BOLD activity in human locus coeruleus. Hum. Brain Mapp. 35, 4140-4154.

Nakane, T., Nihashi, T., Kawai, H., Naganawa, S., 2008. Visualization of neuromelanin in the Substantia nigra and locus ceruleus at $1.5 \mathrm{~T}$ using a 3D-gradient echo sequence with magnetization transfer contrast. Magn. Reson Med. Sci. 7, 205-210.

O'Carroll, R.E., Drysdale, E., Cahill, L., Shajahan, P., Ebmeier, K.P., 1999. Stimulation of the noradrenergic system enhances and blockade reduces memory for emotional material in man. Psychol. Med. 29, 1083-1088.

Ohtsuka, C., Sasaki, M., Konno, K., Koide, M., Kato, K., Takahashi, J., Takahashi, S., Kudo, K., Yamashita, F., Terayama, Y., 2013. Changes in substantia nigra and locus coeruleus in patients with early-stage Parkinson's disease using neuromelanin-sensitive MR imaging. Neurosci. Lett. 541, 93-98.

Ramani, A., Dalton, C., Miller, D.H., Tofts, P.S., Barker, G.J., 2002. Precise estimate of fundamental in-vivo MT parameters in human brain in clinically feasible times. Magn. Reson Imaging 20, 721-731.

Reuter, M., Tisdall, M.D., Qureshi, A., Buckner, R.L., van der Kouwe, A.J., Fischl, B., 2015. Head motion during MRI acquisition reduces gray matter volume and thickness estimates. NeuroImage 107, 107-115.

Ridha, B.H., Symms, M.R., Tozer, D.J., Stockton, K.C., Frost, C., Siddique, M.M., Lewis E.B., MacManus, D.G., Boulby, P.A., Barker, G.J., Rossor, M.N., Fox, N.C., Tofts, P.S. 2007. Magnetization transfer ratio in Alzheimer disease: comparison with volumetric measurements. AJNR Am. J. Neuroradiol. 28, 965-970.

Samuels, E.R., Szabadi, E., 2008. Functional neuroanatomy of the noradrenergic locus coeruleus: its roles in the regulation of arousal and autonomic function part II: physiological and pharmacological manipulations and pathological alterations of locus coeruleus activity in humans. Curr. Neuropharmacol. 6, 254-285.

Sara, S.J., 2009. The locus coeruleus and noradrenergic modulation of cognition. Nat. Rev. Neurosci. 10, 211-223.

Sasaki, M., Shibata, E., Tohyama, K., Takahashi, J., Otsuka, K., Tsuchiya, K., Takahashi, S., Ehara, S., Terayama, Y., Sakai, A., 2006. Neuromelanin magnetic resonance imaging of locus ceruleus and substantia nigra in Parkinson's disease. Neuroreport $17,1215-1218$.

Schwarz, S.T., Xing, Y., Tomar, P., Bajaj, N., Auer, D.P., 2016. In vivo assessment of brainstem depigmentation in Parkinson disease: potential as a severity marker for multicenter studies. Radiology, 160662.

Silvy Collin, L.d.V., Barth, Markus, Fernandez, Guillen, Hermans, Erno, 2014. Phasic BOLD activity in the locus coeruleus and pupil dilation at different levels of tonic arousal, 20th Annual Meeting of the Organization for Human Brain Mapping (OHBM) 2014, Hamburg.

Smythies, J., 1996. On the function of neuromelanin. Proc. Biol. Sci. 263, 487-489.

Sterpenich, V., D'Argembeau, A., Desseilles, M., Balteau, E., Albouy, G., Vandewalle, G., Degueldre, C., Luxen, A., Collette, F., Maquet, P., 2006. The locus ceruleus is involved in the successful retrieval of emotional memories in humans. J. Neurosci. 26, 7416-7423.

Takahashi, J., Shibata, T., Sasaki, M., Kudo, M., Yanezawa, H., Obara, S., Kudo, K., Ito, K., Yamashita, F., Terayama, Y., 2015. Detection of changes in the locus coeruleus in patients with mild cognitive impairment and Alzheimer's disease: high-resolution fast spin-echo T1-weighted imaging. Geriatr. Gerontol. Int. 15, 334-340.

Trujillo, P., Smith, A.K., Summers, P.E., Mainardi, L.M., Cerutti, S., Smith, S.A., Costa A., 2015. High-resolution quantitative imaging of the substantia nigra, Engineering in Medicine and Biology Society (EMBC), 2015 37th Annual International Conference of the IEEE, pp. 5428-5431.

Trujillo, P., Summers, P.E., Ferrari, E., Zucca, F.A., Sturini, M., Mainardi, L.T., Cerutti, S. Smith, A.K., Smith, S.A., Zecca, L., Costa, A., 2016. Contrast mechanisms associated with neuromelanin-MRI. Magn. Reson Med.

Uğurbil, K., 2014. Magnetic resonance imaging at ultrahigh fields. IEEE Trans. Bio Med. Eng. 61, 1364-1379.

Weiss, J.M., Stout, J.C., Aaron, M.F., Quan, N., Owens, M.J., Butler, P.D., Nemeroff, C.B. 1994. Depression and anxiety: role of the locus coeruleus and corticotropinreleasing factor. Brain Res. Bull. 35, 561-572.

Zecca, L., Fariello, R., Riederer, P., Sulzer, D., Gatti, A., Tampellini, D., 2002. The absolute concentration of nigral neuromelanin, assayed by a new sensitive method, increases throughout the life and is dramatically decreased in Parkinson's disease. FEBS Lett. 510, 216-220.

Zecca, L., Stroppolo, A., Gatti, A., Tampellini, D., Toscani, M., Gallorini, M., Giaveri, G. Arosio, P., Santambrogio, P., Fariello, R.G., Karatekin, E., Kleinman, M.H., Turro, N., Hornykiewicz, O., Zucca, F.A., 2004. The role of iron and copper molecules in the neuronal vulnerability of locus coeruleus and substantia nigra during aging. Proc. Natl. Acad. Sci. U. S. A. 101, 9843-9848.

Zecca, L., Swartz, H.M., 1993. Total and paramagnetic metals in human substantia nigra and its neuromelanin. J. Neural Transm. Park Dis. Dement. Sect. 5, 203-213.

Zucca, F.A., Bellei, C., Giannelli, S., Terreni, M.R., Gallorini, M., Rizzio, E., Pezzoli, G., Albertini, A., Zecca, L., 2006. Neuromelanin and iron in human locus coeruleus and substantia nigra during aging: consequences for neuronal vulnerability. J. Neural Transm. 113, 757-767. 